\title{
Image-based Visual/Force/Position Control under Parametric Uncertainties of Camera and Robot Arm
}

\author{
†E.C. Dean-León, †V. Parra-Vega, §A.Espinosa-Romero \\ †Mechatronics Division - CINVESTAV, México, D.F., México, Enail: edean@imail.cinvestav.mx \\ †Center for Advanced Technology - CIATEQ, Retablo 150, Fovissste, Querétaro, Qro. \\ 76150 México Email: vicente.parra@ciateq.mx \\ $\S$ Faculty of Matematics, Universidad Autónoma de Yucatán, México. \\ Email: eromero@tunku.uady.mx.
}

\begin{abstract}
The problem of control law syntheses for constrained dynamic robot manipulators driven by image-based camera information is the subject of this paper. The tasks is to drive the end effector over a constrained surface, while simultaneously controlling the applied force over its normal. This tasks can be found in a variety of applications in the industrial floor, wherein it is reasonable to assume that the exact model of the robot and the camera are unknown. Therefore, the problems is tantamount since the it exposes a synergy of uncalibrated image-based vision servoing schemes with robust force-position control schemes, and all this constitutes a new open paradigm in robotics. In this paper, based on novel and recent robot controllers proposed by the authors, a control system that solves formally the problem above is presented based on multisensor fusion in orthogonalized spaces that decouple the force control task to the image-based visual servoing position task, assuming that the contact point is frictionless. We prove formally the exponential convergence of image-based position and force tracking errors without any knowledge of inertial robot parameters, and simulations allow us to visualize this stability properties under uncertainty of robot kinematic and camera parameters.
\end{abstract}

Index Terms- Visual Servoing, Force Control, Sensor Fusion, Unknown Jacobian.

\section{Introduction}

Multisensor-based ${ }^{1}$ robot control approaches are challenging but may offers a solution to very important and relevant problems in modern robotics, such as those robot tasks that involve joint encoders, force sensors, CCD cameras, range finders, proximity sensors, haptic interfaces, and tactual devices. In order to achieve this sensor fusion-based controller, a careful analysis of the dynamics, sensors behavior, and tasks are required. In this paper, we explore the problem of

\footnotetext{
${ }^{1}$ Sensor fusion deals with the synthesis of signals of different nature from different physical source.
}

controlling uncertain robot manipulators interacting to a rigid environment, controlled by uncalibrated visual feedback signals, joint encoders and force sensors. The sensor signals most provide information that extends the robot work field with nonredundant sensor signals. Also the control law design has to take into account uncertainties of parameters that are intimately related to the camera, such as intrinsic and extrinsic parameters.

\subsection{The problem}

In constrained robot tasks, the end-effector tracks a trajectory over smooth rigid surface, and at the same time, applies a desired normal contact force, see Figure 1. However, when robot dynamics are unknown, it is difficult to keep contact, and when the robot is driven with poorly calibrated visual feedback, it stands as a hard problem to solve. A control scheme that relies of

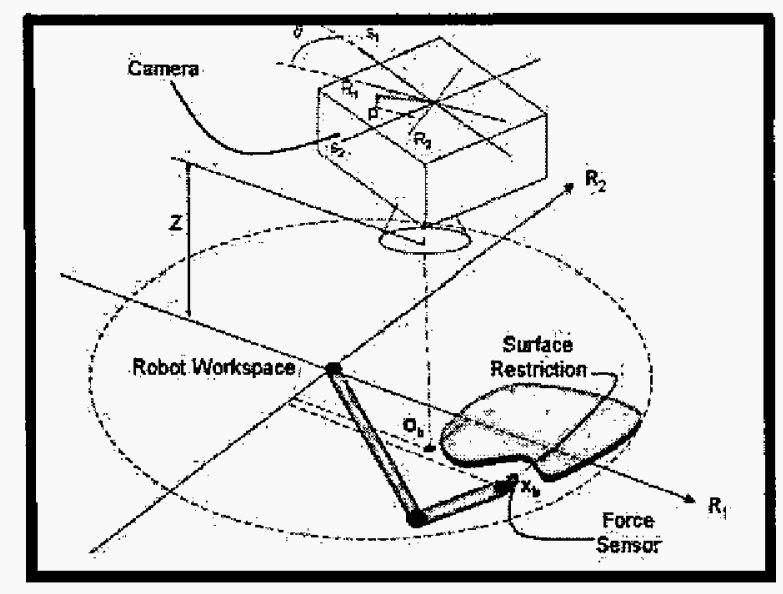

Figure 1: Robot-Force-Vision System.

exact values of the system parameters is always prone to errors in practice, since it is really hard to know exactly the parameters, therefore the control strategy must be robust with respect to robot parametric uncertainties and camera parameters.

A hybrid image-based robust servo visual-force 
scheme is proposed by first time in the literature. The system guarantees exponential tracking of position and force trajectories without any knowledge of robot dynamics and subject to uncertainties of intrinsic and extrinsic camera parameters. This scheme requires low computational cost and presents formal stability proofs. Simulations allows to visualize the closed loop performance using parameters from a real robot and camera system.

\section{Background}

\subsection{Vision/Force Control}

Good sensing abilities of relevant variables are essential to gain higher flexibility and autonomy of robots in a unknown working environment. Often, either vision or force sensor are used, and few vision plus force hybrid schemes have been proposed due to its complexity [J. Baeten, 2000], [A. Namiki, 1999], [S. Jorg, 2000]. Notice that none of these references show robustness to uncertainties, neither robot parameters nor camera parameters, and more importantly, none of them show formal stability results. On the other side, the authors Xiao et al. [Xiao, 2000], present an interesting, but very difficult, scheme of hybrid vision force control in an uncalibrated environment, however this approach includes directly in its synthesis the robot dynamics. In this paper, we extend [E.C Dean, 2002] and synergetically include a force subspace control. The closed-loop system then depends on 3 different sources of sensors: joint encoders for position measurements, force sensor for contact measurements, and a CCD camera for imagebased measurements.

\subsection{Force Control}

Our recent results on force tracking with the model are based on the orthogonalization principle to decouple the position-velocity subspace from the forcemomentum subspace, however these schemes have not been extended or combined beyond constrained robots with state feedback.

\subsection{Visual Servoing}

Control of robots with visual information is often referred as visual servoing. Certainly this is an ill-posed control scheme because the information from camera is not an state of the system, and thus cannot be modified directly by the input of the system as we can see in Weiss et al. [L.Weiss, 1987], [A.Castano, 1994], [N. Papanikolopoulus, 1993] and none of the above works are suitable to establish and maintain contact to a surface. The authors Castano and Hutchinson [A.Castano, 1994], introduce a new technique called visual compliance that is achieved by a hybrid vision/position control structure. In 2002, the authors of this paper [E.C Dean, 2002] proposed a novel scheme based in second order sliding modes to achieve visual servoing with uncalibrated camera, and assume that robot dynamics are unknown.

\section{Nonlinear Robot Dynamics}

\subsection{Robot Direct Kinematics and Dif- ferential Kinematics}

Direct kinematics of a serial $n$-link rigid robot manipulator is given by

$$
x_{b}=f(q)
$$

where $x_{b} \in \Re^{n}$ represents the position of robot end effector in operational workspace, in our case the cartesian space, $q \in \Re^{n}$ is the vector of generalized joint displacements, with finally $f(\cdot): \Re^{n} \rightarrow \Re^{n}$. The differential kinematics of robot manipulator relates velocities in operational space to joint space is defined as follows

$$
\dot{x}_{b}=J(q) \dot{q}
$$

Notice that the analytical jacobian $J(q)$ maps joint velocities to robot cartesian velocities.

\subsection{Camera Model}

\subsubsection{Direct Visual Kinematics.}

The visual position $x_{s} \in \Re^{2}$ of robot end effector in image space (screen) is given by

$$
x_{s}=\alpha R x_{b}+\beta
$$

where $\alpha$ is the scale factor ${ }^{2}, R \in R^{2 \times 2}$ define the matrix $R_{\theta} \in S O(3)$, and $\beta$ is a vector that depends on intrinsic camera parameters.

\subsection{Differential Visual Kinematics}

The differential camera model is then

$$
\dot{x}_{s}=\alpha R \dot{x}_{b}
$$

Using equation $(1) \sim 6$, equation (4) becomes

$$
\dot{x}_{s}=\alpha R J(q) \dot{q}
$$

where $\dot{x}_{s} \in \Re^{2}$ determines the visual robot end effector velocity.

\subsection{Inverse Differential Visual Kine- matics.}

Solving equation (5) for $\dot{q}$, we have the inverse differential kinematics for robot manipulator in terms of visual velocities

$$
\dot{q}=J_{R i n v} \dot{x}_{s}
$$

where, the simplification $J_{R i n v}=J(q)^{-1} R^{-1} \alpha^{-1}=$ $J(q)^{-1} R_{\alpha}^{-1}$, has been used. With $J_{R i n v} \in \Re^{n \times n}$ whose entries are function of robot and camera parameters.

\footnotetext{
${ }^{2}$ Without lose of generality, $\alpha_{0}$ can be considered as a scalar matrix $2 \times 2$.
} 


\subsection{Constrained Robot Dynamics Model.}

The differential algebraic equations that models the constrained robot dynamics are

$$
\begin{aligned}
H(q) \ddot{q}+C(q, \dot{q}) \dot{q}+g(q) & =\tau+J_{\varphi}^{T}(q) f \\
\varphi(x, w) & =0
\end{aligned}
$$

where $H(q) \in R^{n \times n}$ is the symmetric positive definite manipulator inertia matrix, $C(q, \dot{q}) \dot{q} \in R^{n}$ stands for the vector of centripetal and Coriolis torques, $g(q) \in$ $R^{n}$ is the vector of gravitational torques, and finally $f \in R^{n}$ stands for the contact force vector, and $\varphi(x, w)$ is the kinematic restriction, as a rigid surface, where, $\varphi=(x, w): \Omega^{n} \rightarrow \Re$ is a given scalar function, with $x=\left(x_{1}, x_{2}, x_{3}\right)^{T}$ denoting a position of a fixed coordinated system and $w=\left(w_{1}, w_{2}, w_{3}\right)^{T}$ its associated Euler angles. Since the surface is rigid, frictionless and smooth (with continuous gradient), the contact force is given by $f=\left\{(\partial \varphi(x) / \partial x)^{T} /\|\partial \varphi(x) / \partial x\|\right\} \lambda$, with $\lambda \in \Re$ as force magnitude and we introduce the (normalized) constrained Jacobian as $J_{\varphi+}^{T}(q)=$ $(\partial \varphi(x) / \partial x)^{T} /\|\partial \varphi(x) / \partial x\| \subset \Re^{n}$ is a unit vector and stands for contact force direction. Thus, equation (7) can be represented as

$$
H(q) \ddot{q}+C(q, \dot{q}) \dot{q}+g(q)=\tau+J_{\varphi+}^{T}(q) \lambda
$$

Adding and subtracting $H(q) \ddot{q}+C(q, \dot{q}) \dot{q}+g(q)=Y_{b} \theta_{b}$ to (9), we have the following open loop error equation

$$
H(q) \dot{S}_{q}=\tau+J_{\varphi}^{T}(q) \lambda-Y_{r} \theta_{b}-C(q, \dot{q}) S_{q}
$$

with the extended joint error surface $S$ defined with

$$
S_{q}=\dot{q}-\dot{q}_{\tau}
$$

where $\dot{q}_{r}$ is a nominal reference of joint velocities.

\section{Open Loop Error Equation}

\subsection{Orthogonalization Principle.}

Since $\varphi(q)=0 \forall t$, then its time derivative yields

$$
\frac{d}{d t} \varphi(q)=\frac{\partial \varphi(q)}{\partial q} \frac{d q}{d t} \equiv J_{\varphi}(q) \dot{q}=0
$$

This means that $J_{\varphi}(q)$ is orthogonal to $\dot{q}$. That is, $\dot{q}$ belongs to the orthogonal projection matrix $Q$ of $J_{\varphi}(q)$, where

$$
Q=I-\frac{J_{\varphi}}{\left\|J_{\varphi}(x)\right\|^{2}} J_{\varphi}^{T}
$$

Notice that $Q$ spans the tangent plane at the contact point, and in this plane is where exactly $\vec{q}$ lies while the robot end effector is in contact to the constraint surface. Therefore, $J_{\varphi}$ and $Q$ are orthogonal complements since $R^{n}$ is generated by the direct sum of two orthogonal sub-spaces, since $\operatorname{rank}(\mathrm{im}(Q))=$ $m \equiv n-r$ and $\operatorname{rank}\left(\operatorname{im}\left(J_{\varphi}\right)\right)=r$, such as $m+r=n$. In other words,

$$
Q \dot{q}=\dot{q} \quad \text { and } \quad Q J_{\varphi}^{T}=0
$$

These facts are fundamental to solve the problem.

\subsection{Nominal Orthogonalized Refer- ences}

Consider the following

$$
\dot{q}_{r}=\dot{q}_{s}+\dot{q}_{f}
$$

where $\dot{q}_{s}$ and $\dot{q}_{f}$ are to be immersed into orthogonal subspaces, to be able to handle the three source of measurements without mutually conflicting, as it may physically happens. Then, to keep orthogonality, nominal references are to be designed in terms directly of cartesian visual flow and force as follows

$$
\dot{q}_{r}=Q J_{R i n v} \dot{x}_{r}+\beta J_{\varphi}^{T} \dot{q}_{r f}
$$

Now we present $\dot{x}_{r}$ and $\dot{q}_{r f}$.

\subsubsection{Visual position nominal reference.}

Using definition of equation (6) and (13), consider

$$
\dot{x}_{r}=\dot{x}_{s d}-\alpha \Delta x_{s}+S_{s d}-\gamma_{s} \int \operatorname{sign}\left(S_{s \delta}\right)
$$

where $\dot{x}_{s d}$ stands for desired visual trajectory velocity and $\Delta x_{s}=x_{s}-x_{s d}$, is the visual position error, and $S_{s}=\Delta \dot{x}_{s}+\alpha \Delta x_{s}, S_{s d}=S_{s}\left(t_{0}\right) e^{-\kappa_{s} t}, S_{s \delta}=S_{s}-S_{s d}$ with $\Delta \dot{x}_{s}=\dot{x}_{s}-\dot{x}_{s d}$, defines visual velocity error, $\kappa_{s}$ is a positive constant and matrix $\gamma_{s}$ is a matrix of proper dimension, the function sign $(y)$ stands for sign function of vector $y$, and finally $\int \operatorname{sign}(Z) \equiv$ $\int_{t_{0}}^{t} \operatorname{sign}(Z(\zeta)) d \zeta$.

\subsubsection{Force nominal reference.}

Let the nominal force reference be

$$
\dot{q}_{r f}=\Delta F-S_{d F}+\gamma_{F} \int \operatorname{sign}\left(S_{F \delta}\right)
$$

for $S_{F}=\Delta F, S_{F d}=S_{F}\left(t_{0}\right) e^{-\kappa_{F} t}, S_{F \delta}=S_{F}-S_{F d}$, with $\Delta F=\int_{t_{0}}^{t} \Delta \lambda(\zeta) d \zeta, \Delta \lambda=\lambda-\lambda_{d}$, and $\lambda_{d}$ : is the desired contact force, $\kappa_{F}$ is a positive constant, $\gamma_{F}, \beta$ are matrix of proper dimensions.

\subsection{Joint Error Surface}

Using equations (15), (16) y (17) into (11), by using (6), $S_{q}=Q \dot{q}-\dot{q}_{r}$ becomes the following orthogonalized joint error visual/force surface

$$
S_{q}=Q J_{R i n v} S_{v s}-\beta J_{\varphi}^{T} S_{v F}
$$

where

$S_{v s}=S_{s \delta}+\gamma_{s} \int \operatorname{sign}\left(S_{s \delta}\right), S_{v F}=S_{F \delta}+\gamma_{F} \int \operatorname{sign}\left(S_{F \delta}\right)$

Remark 1. The equation(18) assumes exact knowledge of $J_{\text {Rinv }}$, which is unreal for practical applications. Now, we derive a non calibrated (18). 


\subsection{Uncalibrated Joint Error Surface}

In order to model (18) under uncertainties of Jacobian $J(q)$ and visual transformation matrix $R_{\alpha}(\theta)$, we define a new non calibrated nominal reference as follows

$$
\widehat{\dot{q}}_{r}=Q \widehat{J_{R i n i}} \dot{x}_{r}+\beta J_{\varphi}^{T} \dot{q}_{r f}
$$

with $\widehat{J_{R i n v}}$ is an estimated of $J_{R i n v}$, such as rank $J^{-1}(q)$ and $R_{\alpha}^{-1}(\theta)$ is full rank $\forall q \in \Omega$, where $\Omega=$ $\{q \mid \operatorname{rank}(J(q))=n\}$, and $\forall \theta \in \Re$, thus, substituting above equation in (11), we have the uncalibrated joint error surface

$$
\hat{S}_{q}=Q J_{R i n v} \dot{x}_{s}-Q \widehat{J_{R i n v}} \dot{x}_{r}-\beta J_{\varphi}^{T} \dot{q}_{r f}
$$

where $\hat{S}_{q}$ is available because $\dot{q}$ and $\hat{q}_{r}$ are available.

\subsection{Uncertaint Open Loop Equation}

Using (19), the uncertain parametrization $Y_{b} \theta_{b}$ is

$$
H(q) \hat{\dot{q}_{r}}+C(q, \dot{q}) \hat{\dot{q}_{r}}+g(q)=\hat{Y}_{b} \theta_{b}
$$

Adding and subtracting (21) to (9) we obtain

$$
H(q) \widehat{S_{q}}=\tau+J_{\varphi}^{T}(q) \lambda-\hat{Y}_{r} \theta_{b}-C(q, \dot{q}) \widehat{S_{q}}
$$

Now we are ready to present the main result.

\section{Control Law Design}

Theorem 1 Assume that initial conditions and desired trajectories are within the space free of singularities configurations, and consider the robot dynamics (9) in closed loop with the next control law:

$$
\begin{gathered}
\tau=-K_{d} \hat{S}_{q}+J_{\varphi}^{T}(q)\left[-\lambda_{d}+\eta \Delta F\right]+\gamma_{F} J_{\varphi}^{T}(q) * \\
{\left[\tanh \left(\mu S_{q F}\right)+\eta \int \operatorname{sgn}\left(s_{F \delta}\right)\right]}
\end{gathered}
$$

where $K_{d} \in R^{n \times n}$ as a positive symmetric matrix. If $K_{d}$ is large enough and errors of initial conditions are small enough, and if $\gamma_{s} \geq\left\|\frac{d}{d t}\left\{R_{\alpha}(\theta) J(q)\left[\hat{S}_{q}+\left(\widehat{J_{R i n v}}-J_{\text {Rinv }}\right) \dot{x}_{r}\right]\right\}\right\|$ and $\gamma_{F} \geq\left\|\frac{d}{d t}\left[\left(J_{\varphi} J_{\varphi}^{T}(q)\right)^{-1} J_{\varphi} \hat{S}_{q}\right]\right\|$, then exponential convergence of visual and force tracking errors is guaranteed, with a smooth controller.

Proof: The closed loop dynamics between (23) and (22) yields

$$
\begin{gathered}
H(q) \widehat{\dot{S}_{q}}=-K_{d} \hat{S}_{q}+J_{\varphi}^{T}(q)\left[\Delta \lambda+\gamma_{F} \tanh \left(\mu S_{q F}\right)\right]+ \\
\eta J_{\varphi}^{T}(q)\left[\Delta F+\gamma_{F} \int \operatorname{sgn}\left(s_{F \delta}\right)\right]- \\
\hat{Y}_{r} \theta_{b}-C(q, \dot{q}) \widehat{S}_{q}
\end{gathered}
$$

Now we prove that above equation, under stated conditions in the theorem, shows exponential convergence of visual position error and force error, without the exact knowledge of parameters in $J_{R i n v}$. The proof is organized in three sections.

Part I. Closed loop trajectories bound. Consider the time derivative of the following Lyapunou function

$$
V=\frac{1}{2}{\widehat{S_{q}}}^{T} H(q) \widehat{S_{q}}+\beta S_{v F}^{T} S_{v F}
$$

along the solutions of (24) as

$$
\dot{V} \leq-\widehat{S}_{q}^{T} K_{d} \widehat{S_{q}}-\eta \beta S_{v F}^{T} S_{v F}-\left\|\widehat{S_{q}}\right\|\|\varepsilon\|
$$

where $\|\varepsilon\|$ is a functional that bounds $\hat{Y}_{r} \theta_{b}$, similarly to [V. Parra-Vega, 2003]). If $K_{d}, \beta$ and $\eta$ are large enough and the initial errors are small enough, we conclude the seminegative definiteness of (26) outside of hyperball $\varepsilon_{0}=\left\{\widehat{S_{q}} \mid \dot{V} \leq 0\right\}$ centered at the origin, such as the following properties of the state of closed loop system arise

$$
\widehat{S_{q}} \in \mathcal{L}_{\infty} \rightarrow\left\|\widehat{S_{q}}\right\| \leq \varepsilon_{1}
$$

with $\varepsilon_{1}>0$. Then, $\left(S_{s \delta}, \int \operatorname{sign}\left(S_{s \delta}\right)\right) \in \mathcal{L}_{\infty}$ and since desired trajectories are $C^{2}$ and feedback gains are bounded, we have that $\left(\widehat{\vec{q}}_{r}, \widehat{q}_{r}\right) \in \mathcal{L}_{\infty}$, which implies that $Y_{\text {cont }} \in \mathcal{L}_{\infty}$. The right hand side of (22) shows that $\varepsilon_{2}>0$ exists such that $\left\|\hat{\dot{S}}_{q}\right\| \leq \varepsilon_{2}$. This result shows only local stability of $\widehat{S_{q}}$ and $\widehat{S}_{q}$, now we prove that the sliding modes arises.

Part II. Sliding modes (visual tracking and force). Adding and subtracting $Q J_{R i n v} \dot{x}_{r}$ to (20) we obtain

$$
\hat{S}_{q}=Q\left\{J_{R i n v} S_{v s}-\Delta J_{R i n v} \dot{x}_{r}\right\}-\beta J_{\varphi}^{T}\left\{S_{v F}\right\}
$$

where $\Delta J_{\text {Rinv }}=\widehat{J_{R i n v}}-J_{\text {Rinv }}$. Since $\hat{S}_{q} \in \mathcal{L}_{2}$, and $J_{R i n v}$ and $Q$ are bounded (is easily to prove that if $J(q)$ is bounded then $J_{R i n v}$ is bounded), then $Q J_{R i n v} S_{v s}$ is bounded and, due to $\varphi(q)$ is smooth and lies in the reachable robot space and $S_{v F} \rightarrow 0$, then $\beta J_{\varphi}^{T} S_{u F} \rightarrow 0$. Now, taking into account that $\widehat{\dot{S}_{q}}$ is bounded, then $\frac{d}{d t} J_{R i n v} Q S_{v s}$ and $\frac{d}{d t} \beta J_{\varphi}^{T} S_{v F}$ are bounded (this is possible because $J_{\varphi}^{T}$ is bounded and so $\dot{Q}$ is). All this chanin of conclusions proves that there exists constants $\varepsilon_{3}>0$ and $\varepsilon_{4}>0$ such that

$$
\left|\dot{S}_{v s}\right|<\varepsilon_{3}, \quad\left|\dot{S}_{v F}\right|<\varepsilon_{4}
$$

Now, we have to prove that for a proper $\gamma_{s}$ and $\gamma_{F}$, we can conclude that trajectories of visual position and force converges to zero. This is possible through sliding modes for the subspace of visual position $Q$ and the subspace of force $J_{\varphi}^{T}(q)$. Considering that operator $Q J_{\text {Rinv }}$ spans the vector $\hat{S}_{q}$ in its image $i m\left\{Q J_{R i n v}\left(S_{v s}\right)\right\} \equiv S_{v s}^{i m}$ and the operator $\beta J_{\varphi}^{T}$ spans 
the same vector in its image $i m\left\{\beta J_{\psi}^{T}\left(S_{v F}\right)\right\} \equiv S_{v F}^{i m}$, this implies that

$$
\begin{aligned}
\hat{S}_{q} & =Q\left\{J_{R i n v} S_{v s}-\Delta J_{R i n v} \dot{x}_{r}\right\}-\beta J_{\varphi}^{T}\left\{S_{v F}\right\} \\
& =S_{v s}^{i m}-i m\left\{\Delta J_{R i n v} \dot{x}_{T}\right\}-S_{v F}^{i m}
\end{aligned}
$$

where $S_{v s}^{i m}-i m\left\{\Delta J_{R i n v} \dot{x}_{r}\right\}$ and $S_{v F}^{i m}$ belongs to a orthogonal complements, that means $\left\langle S_{v s}^{i m}-i m\left\{\Delta J_{R i n v} \dot{x}_{r}\right\}, S_{v F}^{i m}\right\rangle=0$. We are able to analyze the $S_{v s}^{i m}-i m\left\{\Delta J_{R i n v} \dot{x}_{r}\right\}$ dynamics, independently of $S_{v F}^{i m}$, since $S_{v F}^{i m}$ belongs to the kernel of $Q$. This is verified if we multiply (29) for $Q^{T}$,

$$
\begin{aligned}
Q^{T} \hat{S}_{q} & =Q^{T} Q\left\{J_{\text {Rinv }} S_{v s}-\Delta J_{R i n v} \dot{x}_{r}\right\}-\underbrace{\beta Q^{T} J_{\varphi}^{T}\left\{S_{v F}\right\}}_{\text {equal } 0} \\
& =S_{v s}^{i m}-i m\left\{\Delta J_{R i n v}, \dot{x}_{r}\right\}
\end{aligned}
$$

since $Q$ is idempotent $\left(Q^{T} Q=Q\right)$. It is important notice that if $A x=A y$ for any square matrix $A$ and any couple of vectors $x, y$, then $x=y$. Thus, the equation (30) means that for the subspace $Q$, the equality $\hat{S}_{q}=Q\left\{J_{R i n v} S_{v s}-\Delta J_{R i n} \dot{x}_{r}\right\}$ is valid within span of $Q$. Now, if we multiply $\hat{S}_{q}$ for $J_{\varphi}$ we have:

$$
\begin{aligned}
J_{\varphi} \hat{S}_{q} & =\underbrace{J_{\varphi} Q\left\{J_{\text {Rinv }} S_{v s}-\Delta J_{\text {Rinv }} \dot{x}_{r}\right\}}_{\text {equal } 0}-\beta J_{\varphi} J_{\varphi}^{T}\left\{S_{v F}\right\} \\
& =S_{v F}^{\text {im }}
\end{aligned}
$$

Part II.a: Exponential convergence of visual tracking errors. According to $Q^{T} \hat{S}_{q}=$ $Q\left\{J_{R i n v} S_{v s}-\Delta J_{R i n v} \dot{x}_{r}\right\}$ then $\hat{S}_{q} \equiv J_{R i n v} S_{y s}-$ $\Delta J_{R i n} \dot{x}_{r}$ in the subspace image of $Q$, however notice that $Q$ is not full rank, then this equality is valid locally, not globally. In this local neighborhood, if we multiply the equality $\hat{S}_{q}=Q\left\{J_{R i n v} S_{v s}-\Delta J_{R i n v} \dot{x}_{r}\right\}$ by $R_{\alpha}(\theta) J(q)\left(J_{R i n v}=J^{-1}(q) R_{\alpha}^{-1}(\theta)\right)$, we have

$$
\begin{array}{r}
R_{\alpha}(\theta) J(q) \hat{S}_{q}=S_{s \delta}+\gamma_{s} \int \operatorname{sign}\left(S_{s \delta}\right)- \\
R_{\alpha}(\theta) J(q)\left\{\Delta J_{R i n v} \dot{x}_{r}\right\}
\end{array}
$$

Taking the time derivative of the above equation, and multiply it by $S_{s \delta}^{T}$ produces

$$
\begin{aligned}
S_{s \delta}^{T} \dot{S}_{s \delta} & =-\gamma_{s} S_{s \delta}^{T} \operatorname{sign}\left(S_{s \dot{b}}\right)+ \\
& S_{s \delta}^{T} \frac{d}{d t}\left[R_{c}(\theta) J(q)\left(\hat{S}_{q}+\Delta J_{R i n v} \dot{x}_{r}\right)\right] \\
& \leq-\mu_{s}\left|S_{s \delta}\right|
\end{aligned}
$$

where $\varepsilon_{5}=\frac{d}{d t}\left[R_{\alpha}(\theta) J(q)\left(\hat{S}_{q}+\Delta J_{R i n v} \dot{x}_{T}\right)\right]$, and $\mu_{s}=\gamma_{s}-\varepsilon_{5}$. Thus, we obtain the sliding condition if

$$
\gamma_{s}>\varepsilon_{5}
$$

such as, $\mu_{s}>0$ of (33) guarantee the sliding mode at $S_{s \delta}=0$ in a time $t_{s}=\frac{\left|S_{s \delta}\left(t_{0}\right)\right|}{\mu_{s}}$. However, notice that for any initial condition $S_{s \delta}\left(t_{0}\right)=0$, then $t_{s}=0$, which implies that the sliding mode at $S_{s \delta}(t)=0$ is guaranteed for all time. Then, we have

$$
S_{s}=S_{s d} \forall t \rightarrow \Delta \dot{x}_{s}=-\alpha \Delta x_{s}+S_{s}\left(t_{0}\right) e^{-\kappa_{s} t}
$$

this implies that the visual tracking errors locally tends to zero exponentialiy fast, this is

$$
x_{s} \rightarrow x_{s d}, \dot{x}_{s} \rightarrow \dot{x}_{s d}
$$

implying that the robot end-effector converges to the desired image $x_{s d}$, with given velocity $\dot{x}_{s d}$.

Part II.b: Exponential convergence of force tracking errors. In the same way, if we multiply (31) for $\left(J_{\varphi} J_{\varphi}^{T}(q)\right)^{-1}$, we obtain

$$
\begin{aligned}
\left(J_{\varphi} J_{\varphi}^{T}(q)\right)^{-1} J_{\varphi} \hat{S}_{q} & =-\beta J_{\varphi} J_{\varphi}^{T}\left\{S_{v F}\right\} \\
J_{\varphi}^{\#}(q) \hat{S}_{q} & =S_{F \delta}+\gamma_{F} \int \operatorname{sign}\left(S_{F \delta}\right)
\end{aligned}
$$

where $J_{\varphi}^{\#}(q)=\left(J_{\varphi} J_{\varphi}^{T}(q)\right)^{-1} J_{\varphi}$. Derivating the above equation and multiply for $S_{F \delta}^{T}$ lies

$$
\begin{aligned}
S_{F \delta}^{T} \dot{S}_{F \delta} & =-\gamma_{F}\left|S_{F \delta}\right|+S_{F \delta}^{T} \frac{d}{d t}\left(J_{\varphi}^{\#}(q) \hat{S}_{q}\right) \\
& \leq-\gamma_{F}\left|S_{F \delta}\right|+\left|S_{F \delta}\right| \varepsilon_{6}
\end{aligned}
$$

where $\varepsilon_{6}=\frac{d}{d t}\left[\left(J_{\varphi} J_{\varphi}^{T}(q)\right)^{-1} J_{\varphi} \hat{S}_{q}\right]$. If $\gamma_{F}>\varepsilon_{6}$, then a siiding mode at $S_{F \delta}(t)=0$ is induced in a time $t_{f} \leq \frac{\left|S_{F \delta}\left(t_{0}\right)\right|}{\mu_{F}}$, but $S_{F \delta}\left(t_{0}\right)=0$, and that means $\Delta F=$ $\Delta F\left(t_{0}\right) e^{-\kappa_{F} t}$. Moreover, in [V. Parra-Vega, 2003] it is showed that the convergence of force tracking errors arises, thus $\lambda \rightarrow \lambda_{d}$ exponentially fast.

Remark 2. We have proved that $J(q)\left(t_{0}\right)$ is not singular for all time, because $J_{R i n v}=J^{-1}(q) R_{\alpha}^{-1}(\theta)$ at a given initial conditions, and because desired trajectories belong to robot workspace $\Omega$, and trajectories converge with an exponential envelope.

\section{Simulations.}

In order to simulate the system the robot and camera parameters are take from a real ones. The desired trajectories for the simulation was $: x_{s}=a R[x c d ; y c d]+$ $\beta, x c d=0.5 ; y c d=0.5+r * \sin (w * t) ; r=0.1, w=$ 0.5 . Figures show the calibrated and uncalibrated cases ( $50 \%$ of parametric uncertainty on $J_{\text {Rinv }}$ ). Feedback gains for the uncalibrated system are $\mathrm{r}_{f}=20$, $\gamma_{f}=3.0, \eta=0.029, \beta=1.0, K_{d}=90, \alpha=40$, $\kappa s=20, \gamma_{s}=7.8$, the calibrated system constants are: $\hbar_{f}=20, \gamma_{f}=3.0, \eta=0.029, \beta=1.0, K_{d}=70, \alpha=$ $25, \kappa s=20, \gamma_{s}=4.02$. The figures fully comply with the stability properties shown in the theorems.

\section{Conclusions}

This paper introduces a new scheme for hybrid image based visual/force servoing for constrained dynamical robot system. It is shown that exponential convergence arises for position-velocity and force subspaces, even when neither robot parameters nor camera parameters nor analytical jacobian are known. A formal proof of stability is given, and simulation results show that the high performance of control law is maintained even under severe parametric uncertainty. 


\section{Acknowledgements}

E. Dean thanks CONACYT support under a doctoral scholarship. V. Parra-Vega carries out this research while he is on a sabbatical leave from Mechatronics Division, CINVESTAV, at the Information Technology Division of CIATEQ. This author acknowledges support from CONACYT project 39727-Y and CUDI project of Internet2 Applications Spring 2003.

\section{References}

[A. Namiki, 1999] A. Namiki, Y. Nakabo, I. I. M. I. (1999). High speed grasping using visual and force feedback. Proceedings of the 1999 IEEE International Conference on Robotics and Automation, Detroit, $M I$, pages $3195-3200$.

[A.Castano, 1994] A.Castano, S. (1994). Visual compliance:task directed visual servo control. IEEE Trans. on Robot. Autom., 10(3):334-342.

[E.C Dean, 2002] E.C Dean, V.Parra-Vega, A. E. (2002). Seguiniento dinámico de robots manipuladores utilizando retroalimentación visual no calibrada. Tesis de Maestría, Departamento de Ingeniería Eléctrica, Sección de Mecatrónica, CINVES$T A V, I P N$.

[J. Baeten, 2000] J. Baeten, W. Verdonck, H. B. J. D. S. (2000). Combining force control and visual servoing for planar contour following. Machine Intelligence and Robotic Control, 2(2):69-75.

[L.Weiss, 1987] L.Weiss, A.Sanderson, C. N. (1987). Dynamicsensor based control of robots with visual feedback. IEEE Journal on Robot. and Autom., $3: 404-417$.

[N. Papanikolopoulus, 1993] N. Papanikolopoulus, P. Khosla, T. K. (1993). Visual tracking of a moving target by a camera mounted on a robot: A combination of control and vision. IEEE $J$. on Robot. and Autom., 9:14-35.

[S. Jorg, 2000] S. Jorg, J. Langwald, J. S. G. H. C. N. (2000). Flexible robot assambly using a multisensory approach. Procc. of the 2000 IEEE Int. Conf. Robot. and Autom., San Francisco, CA, pages 3687-3694.

[V. Parra-Vega, 2003] V. Parra-Vega, S. Arimoto, Y. L. G. H. P. A. (2003). Dynamic sliding PID control for tracking of robot manipulators: Theory and experiments. IEEE Transactions in Robotics and Automation.

[Xiao, 2000] Xiao, D. (2000). Sensor-hybrid Position/Force control of a robot manipulator in an un calibrated enviroment. IEEE Trans. Control Systm Technology, 8(4):635-645.
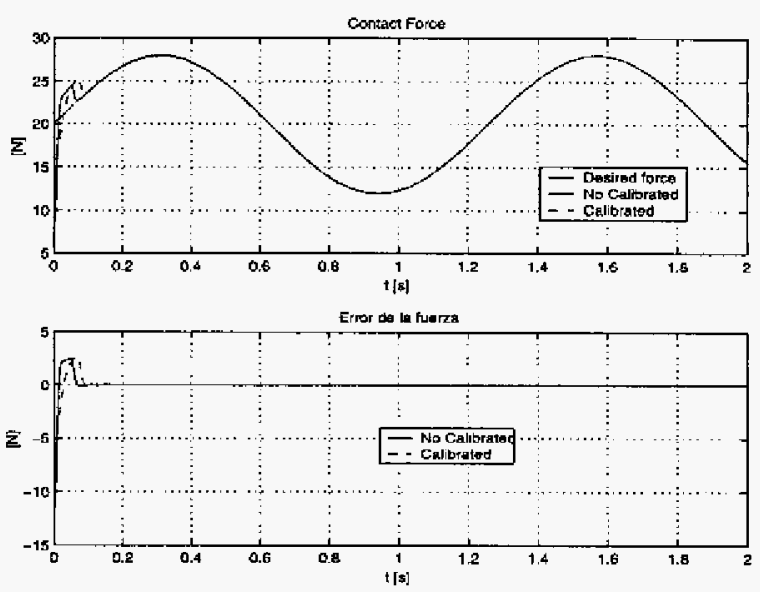

Figure 2: Very fast convergence of force tracking errors, with a short transient due to the uncertainties.
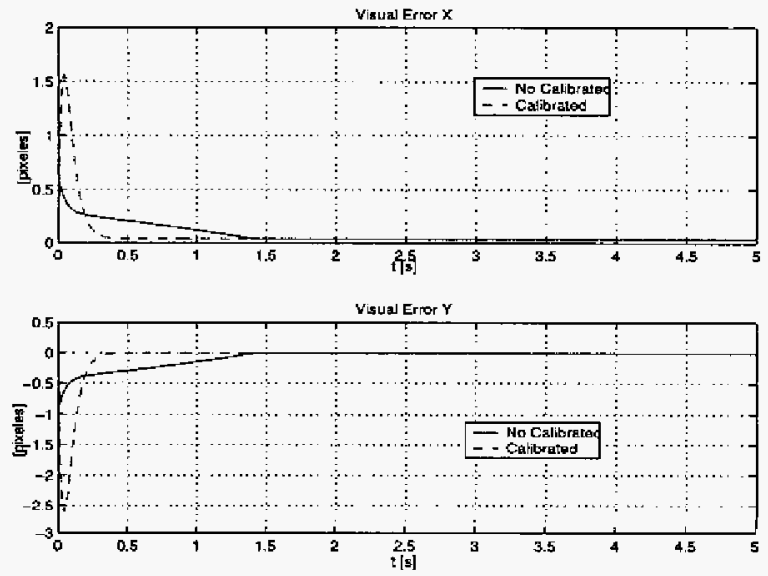

Figure 3: Visual exponential tracking errors.
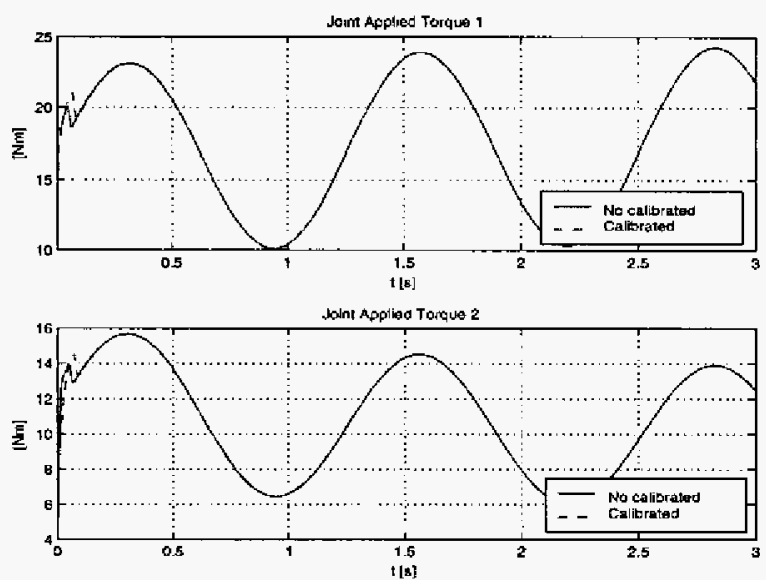

Figure 4: Control input with very smooth activity. 